\title{
Genome-based analysis of Carbapenemase- producing Klebsiella pneumoniae isolates from German hospital patients, 2008-2014
}

\author{
Laura Becker ${ }^{1}$, Martin Kaase ${ }^{2,3}$, Yvonne Pfeifer ${ }^{1}$, Stephan Fuchs ${ }^{1}$, Annicka Reuss ${ }^{1}$, Anja von Laer ${ }^{1}$, Muna Abu Sin ${ }^{1}$, \\ Miriam Korte-Berwanger ${ }^{2,4}$, Sören Gatermann ${ }^{2}$ and Guido Werner ${ }^{1 *}$
}

\begin{abstract}
Background: By using whole genome sequence data we aimed at describing a population snapshot of carbapenemase-producing K. pneumoniae isolated from hospitalized patients in Germany between 2008 and 2014.

Methods: We selected a representative subset of 107 carbapenemase-producing K. pneumoniae clinical isolates possessing the four most prevalent carbapenemase types in Germany (KPC-2, KPC-3, OXA-48, NDM-1). Isolates were processed via illumina NGS. Data were analysed using different SNP-based mapping and de-novo assembly approaches. Relevant information was extracted from NGS data (antibiotic resistance determinants, wzi gene/cps type, virulence genes). NGS data from the present study were also compared with 238 genome data from two previous international studies on K. pneumoniae.

Results: NGS-based analyses revealed a preferred prevalence of KPC-2-producing ST258 and KPC-3-producing ST512 isolates. OXA-48, being the most prevalent carbapenemase type in Germany, was associated with various $K$. pneumoniae strain types; most of them possessing IncL/M plasmid replicons suggesting a preferred dissemination of bla OXA-48 via this well-known plasmid type. Clusters ST15, ST147, ST258, and ST512 demonstrated an intermingled subset structure consisting of German and other European K. pneumoniae isolates. ST23 being the most frequent MLST type in Asia was found only once in Germany. This latter isolate contained an almost complete set of virulence genes and a K1 capsule suggesting occurrence of a hypervirulent ST23 strain producing OXA-48 in Germany.

Conclusions: Our study results suggest prevalence of "classical" K. pneumonaie strain types associated with widely distributed carbapenemase genes such as ST258/KPC-2 or ST512/KPC-3 also in Germany. The finding of a supposed hypervirulent and OXA-48-producing ST23 K. pneumoniae isolates outside Asia is highly worrisome and requires intense molecular surveillance.
\end{abstract}

Keywords: KPC, OXA-48, ST258, Hypermucoviscous, Hypervirulent, ST23, K1 capsule

\section{Background}

Third-generation cephalosporin-resistant and carbapenemresistant Klebsiella pneumoniae are important health-care pathogens associated with increased morbidity and mortality among high-risk patients. Recent data from the European Antimicrobial Resistance Surveillance network (EARS-Net) for health-care associated pathogens (from invasive infections) constitute a rising number of thirdgeneration cephalosporin-resistant and carbapenem-

\footnotetext{
* Correspondence: wernerg@rki.de

${ }^{1}$ Robert Koch Institute, Berlin, Germany

Full list of author information is available at the end of the article
}

resistant K. pneumoniae all over Europe [1]. In the period 2012 to 2015 some countries, such as Spain, Portugal and Croatia, experienced rising trends of carbapenem resistance still at a low level $(<4 \%)$ but triggering the overall European trend. In other countries such as Romania, Italy, and Greece, the rates were already much higher and, in addition, partly showed an increasing trend (Romania). In contrast to this, the majority of European countries, including Germany demonstrated rates below 1\% without showing any significant development in recent years. Thus, Germany could be considered as a low-prevalence country according to EARS-Net data regarding the

(c) The Author(s). 2018 Open Access This article is distributed under the terms of the Creative Commons Attribution 4.0 International License (http://creativecommons.org/licenses/by/4.0/), which permits unrestricted use, distribution, and reproduction in any medium, provided you give appropriate credit to the original author(s) and the source, provide a link to the Creative Commons license, and indicate if changes were made. The Creative Commons Public Domain Dedication waiver (http://creativecommons.org/publicdomain/zero/1.0/) applies to the data made available in this article, unless otherwise stated. 
occurrence and spread of carbapenem-resistant $K$. pneumoniae. However, the National Reference Centre (NRC) for Gram-negative nosocomial pathogens in Germany is challenged by analysing an increasing number of several thousand carbapenem-resistant Enterobacteriaceae, Pseudomonas aeruginosa and Acinetobacter each year, starting with only a few hundred isolates back in 2008/2009. In 2015 and 2016 diagnostic laboratories throughout Germany sent 1148 and 1317 carbapenemresistant $K$. pneumoniae isolates to the NRC, respectively, and exactly $51 \%$ of them possessed a carbapenemase $[2,3]$. The most frequent carbapenemase type in these isolates was OXA-48, followed by KPC-2/- 3 and NDM-1.

Since May 2016, Acinetobacter spp. and Enterobacteriaceae with carbapenem non-susceptibility are mandatorily notifiable to the national health authorities in Germany. From September 2016 to August 2017, 4028 notifications were reported. The most prevalent notified pathogen was K. pneumoniae (1147, 29\%). Notifications of carbapenem-resistant $K$. pneumoniae came from all over Germany with a range of 3 to 267 notifications per federal state. For 293 (26\%) of those, a carbapenemase was reported. The most common specified carbapenemases were OXA-48-like (53\%), KPC-2/-3 (17\%) and NDM-1 (12\%)(A.R., A.v.L., M.A.S., unpublished data).

With this study we aimed at describing a population snapshot of carbapenemase-producing $K$. pneumoniae isolated from hospitalized patients in Germany. We compiled a representative subset of 107 carbapenemaseproducing K. pneumoniae collected between 2008 and 2014 from all over Germany and harbouring carbapenemases OXA-48, KPC-2, KPC-3 and NDM-1. We performed whole genome sequencing to (i) infer the phylogenetic relatedness of corresponding isolates including identification of the multi-locus sequence type (MLST); (ii) extract $\beta$-lactamase- and virulence gene content; (iii) deduce the capsule type (wzi typing); and (iv) reconstruct the plasmid content. Finally, we compared our results with $K$. pneumoniae isolates from clinical and ambulatory settings in Europe and worldwide (carbapenemase-producers were in the minority).

\section{Methods}

\section{Strain collection}

All carbapenemase-producing $K$. pneumoniae isolates included in the study were collected during 2008 to 2014 by the NRC and the Robert Koch Institute (RKI). We selected a comprehensive subset of 107 strains including only carbapenemase-producing $K$. pneumoniae possessing the four most prevalent carbapenemase types in Germany (KPC-2, KPC-3, OXA-48, NDM-1). Isolates were selected based on a collection of 2104 strains at both participating reference institutions (NRC $n=1904$; RKI $n=200$ ) [2]. The selected sample set also contained type strains representing prominent outbreaks (one isolate per setting) [4-7] and other isolates representing previously unpublished outbreaks which were recognized as part of the routine work of the NRC and RKI. The isolate collection covered 12 of the 16 federal states in Germany (Additional file 1: Figure S1 and Additional file 2: Table S1). The number of carbapenemase-producing $K$. pneumoniae isolates sent to the NRC and RKI increased continuously until 2014; however, we tried to balance the selection of isolates over the collection period appropriately (2010, $n=20 ; 2011, n=20$; $2012, n=25 ; 2013, n=22 ; 2014, n=13)$. The distribution of carbapenemase genes within our sample set represents the distribution among the entire set of 2104 clinical carbapenemase-producing $K$. pneumoniae isolates in both reference institutions during the time period analysed (bla $a_{O X A-48} n=42,39 \%$; bla KPC-2 $_{2} n=34 ; 32 \%$; bla KPC-3 $\left.n=26,24 \% ; b_{N D M-1} n=5,5 \%\right)$. Although strain sending to the NRC or the RKI is voluntary, the annual collection is comprehensive enough to deduce a broader picture and allow some general assumptions $[2,8]$.

\section{Whole genome sequencing (WGS)}

Bacteria were grown in Brain Heart Infusion (BHI) broth. DNA was extracted from overnight cultures using the MagAttract Kit (Qiagen, Hilden, Germany) and the DNeasy Blood \& Tissue Kit (Qiagen) in line with the manufacturer's instructions. Qubit dsDNA HS Assay Kit (Invitrogen/ Thermo Fisher Scientific, Karlsruhe, Germany) was used for DNA quantification. Sequencing libraries were prepared applying the Nextera XT Kit (Illumina, San Diego, USA) and sequenced on an Illumina Miseq using v3 chemistry $(2 \times 300 \mathrm{bp})$ according to the manufacturer's protocol.

\section{Mapping-based WGS data analysis}

A base quality-dependent trimming was performed for Illumina raw reads applying Trimmomatic [9](version 0. 32, parameters: illuminaclip off, slidingwindow 4:15, leading 3, trailing 3, crop off, headcrop off, minlen 36, avgqual off) using BWA-SW (version 0.7.13-r1126, default parameters) [10]. Isolates generating a high number of ambiguous sites (cutoff: $\geq 15 \%$ " $N$ " in the consensus sequence) were excluded from the mapping and tree calculation (see also Results chapter). The reference genome was selected using refRank (version 1.0.0; see below). Single nucleotide polymorphisms (SNPs) were called using Varscan v2.3 [11] and variant positions were filtered using SNPfilter (version 2.2.0; see below). Based on the aligned variant positions maximum-likelihood trees were calculated using RAxML (version 8.2.7, model GTR GAMMA, 100 bootstraps) [12]. Artificial Illumina reads were generated from the FASTA-sequence downloaded from NCBI using ART (version 2.5.8, parameters: system MiSeq v3, read length 250, paired end, fragment length 600, standard deviation 300, coverage 100) [13] 
and mapped to the reference genome as described above for the sequenced isolates.

\section{Reference genome selection using refRank}

To optimize the selection of a reference sequence for next generation sequencing (NGS) read alignment (mapping), we created a Python-based application called refRank, which provides a coverage-based reference ranking. For this purpose, datasets of paired-end or unpaired reads are aligned against a collection of defined reference sequences using BWA-SW [10] or BWA-MEM (arXiv:1303.3997v2 [q-bio.GN]). Computational costs can be reduced by using only a fraction of randomly picked (paired-end) reads of each dataset (e.g. 10\% of all reads). Per base coverage is then determined using SAMtools [14] and normalized to the reference length and the number of total (mapped and unmapped) reads according to a detached formula 1 described in [15]. Dataset-specific reference ranking is based on the calculated $\mathrm{C}$ scores. Additionally, a global reference ranking based on all datasets is provided by calculating the grand average of reference-specific $\mathrm{C}$ scores. The source code for refRank is freely available under the terms of the GNU (https://www.gnu.org/) General Public License v3.0 from https://gitlab.com/s.fuchs/refRank.

In the present study, refRank (version 1.0.0) was used with default parameters. The entire collection of completed genomic sequences of K. pneumoniae $(n=79)$ available on RefSeq (accessed on 1st Dec 2016) was used as reference dataset. All 105 raw read datasets were trimmed using Trimmomatic (see above) $[9,16]$. According to the reference ranking (not shown), the genomic sequence of K. pneumoniae KPNIH30 (NZ_CP009872.1) has been selected as a reference for further analysis.

\section{Variant site filtering using SNPfilter}

A python-based application called SNPfilter was developed to condense the alignment of all reconstructed sequences to variant positions only and, thus, to significantly reduce computational costs of subsequent phylogenetic analyses. Optionally, sites can be excluded based on (i.) ambiguous base calls and/or deletions; (ii.) SNP accumulation (based on exclusion distance); and (iii.) user-defined regions (based on genomic coordinates). Importantly, circular replicon topologies can be considered when applying an exclusion distance for accumulated variant positions. The output of SNPfilter provides different files containing (i.) aligned variant sites that meet the filter criteria (FASTA format); (ii.) general information such as sequence names, number of sites containing ambiguous base calls or gaps, number of variants before and after filtering (TXT format); and (iii.) coordinate-specific filter status and sequence information (CSV format). The source code for snpFilter is freely available under the terms of the GNU General Public License v3.0 from https://gitlab.com/s.fuchs/snpFilter [15].

In the present study sites containing gaps or ambiguous base calls were excluded. To exclude SNPs in repetitive regions and phage sequences, the reference sequence $K$. pneumoniae KPNIH30 (NZ_CP009872.1) was analysed using the repeat analysis tool in Kodon (Applied Maths) version 3.62 and the phage search tool PHASTER [16]. Additionally, SNPs in regions of annotated phages and transposases were rejected (not shown). Trees were illustrated using FigTree v1.4.3. (http://tree.bio.ed.ac.uk/software/figtree/; last access: 25/04/2018) and iTOL v3 (https://itol.embl.de/; last access: 25/04/2018).

\section{De novo assembly}

Raw reads were trimmed applying Trimmomatic 16 (version 0.32, parameters: Illuminaclip off, maxinfo 50:0.8, leading 3, trailing 3, crop off, headcrop off, minlen 36 , avgqual off). Trimmed reads were de novo assembled using A5-miseq (version 20,150,522) [17].

\section{WGS-based deduction of MLST, wzi type, $\beta$-lactamase and virulence genes}

WGS data of all isolates were analysed using Resfinder [18], PlasmidFinder and the MLST tool [19] provided by the Centre for Genomic Epidemiology (https://cge.cbs. dtu.dk/services/; last access: 25/04/2018). Determination of capsular type for Klebsiella strains was conducted by $w z i$ gene sequencing extracted from contigs derived from de novo assembly [20] and using the websites of the Pasteur Institute (http://bigsdb.pasteur.fr/perl/ bigsdb/bigsdb.pl?db=pubmlst_klebsiella_seqdef_public\&page $=$ downloadAlleles\&scheme_id $=4 \&$ render $=1$; last ac cess 25/04/2018). Furthermore, all sequenced genomes were screened for the presence of the following virulence genes or gene clusters using the reference databank of the Pasteur Institute: $m r k$, yersiniabactin, allantionase, colibactin, salmochelin, aerobactin, $k f u$, microcin, $r m p A, r m p A 2$, and $k v g$. Genes with mismatches or incomplete sequences were additionally analysed in Geneious searching for Stopp codons and inserted IS elements (Geneious v10, Biomatters Lmtd., New Zealand). Primers used for additional and confirmatory PCR amplification of different virulence and $\beta$-lactamase genes are given as a Additional file 3: Table S2.

\section{Phenotypic test for hypervirulence}

In order to test for a putative hypervirulence or hypermucoviscous phenotype, we established the so-called 'string test' [21]. The hypervirulent $K$. pneumoniae isolate of ST2398 possessing a K2 capsule was used as a positive control [22]. 


\section{Results}

\section{MLST analyses}

We determined the $K$. pneumoniae MLST types (STs) and the carbapenemase genes from the WGS data. The 107 sequenced isolates belonged to 21 different STs. Figure 1 shows the distribution of STs among the four analysed carbapenemase types (OXA-48, KPC-2, KPC-3, NDM-1). The majority of KPC-2-producing isolates belonged to ST258 $(n=27,80 \%)$. Three isolates were ST147 and one each belonged to ST101, ST340, ST347 and ST784. KPC-3-producing isolates demonstrated a low diversity; 21 isolates (81\%) were ST512, four were ST258 and one isolate was ST37. Altogether 42 OXA48-producing isolates revealed 15 different STs, whereas 15 isolates (36\%) were ST101 and nine (21\%) were ST147. The other STs appeared less than four times. Furthermore, one new ST (ST2254; allelic profile: gapA _25, infB_33, mdh_141, pgi_26, phoE_7, rpoB_1, tonB_ $56)$ with the carbapenemase gene $b l a_{\text {OXA-48 }}$ was identified. The five NDM-1-producers that were included in this study could be assigned to four different STs (ST15, ST16, ST147 and ST340).

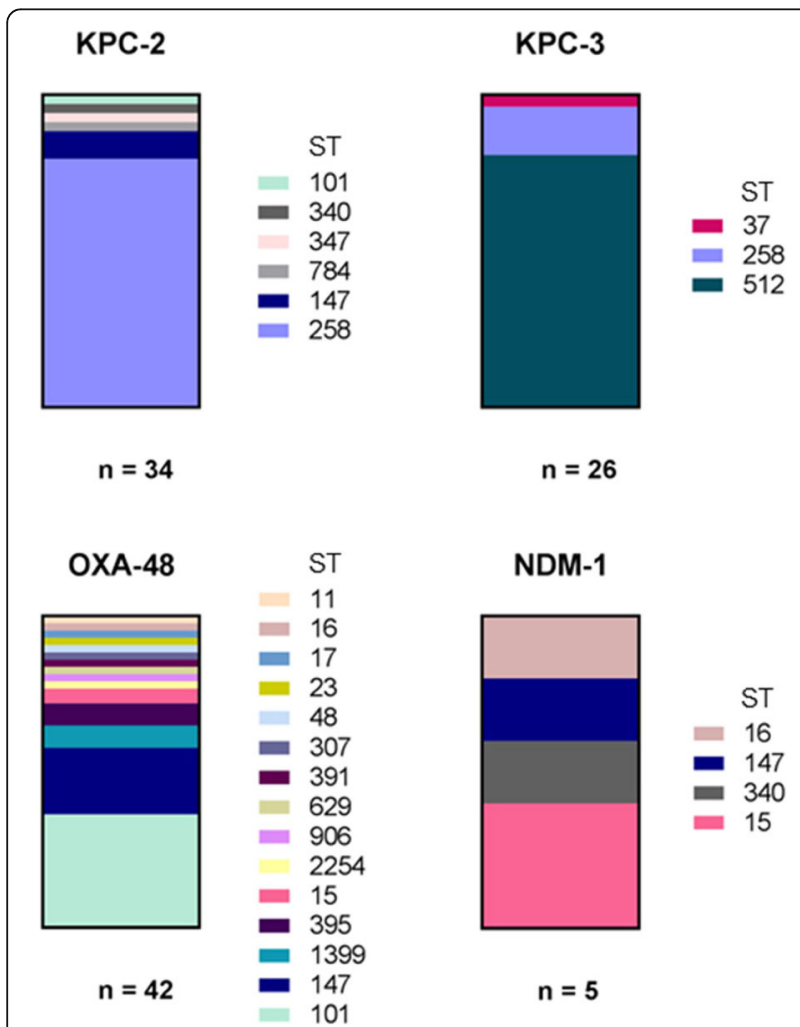

Fig. 1 Distribution of Klebsiella-MLST and carbapenemase types. The columns show the relative amount of corresponding sequence type (ST) within the group of carbapenemase-producing isolates (KPC-2, KPC-3, OXA-48, NDM-1)
Table 1 Additional $\beta$-lactamase genes in carbapenemaseproducing $K$. pneumoniae isolates. Counts are given in exact numbers and in percentages. Genes were extracted from NGS data using ResFinder software

\begin{tabular}{|c|c|c|c|c|c|}
\hline & $\mathrm{KPC}-2$ & $\mathrm{KPC}-3$ & OXA-48 & NDM-1 & Total \\
\hline & $n=34$ & $n=26$ & $n=42$ & $n=5$ & $n=107$ \\
\hline \multicolumn{6}{|c|}{ Chromosomal $\beta$-lactamase genes } \\
\hline$b / a_{\mathrm{SHV}-1}$ & $2(6 \%)$ & - & $16(38 \%)$ & $1(20 \%)$ & $19(18 \%)$ \\
\hline$b / a_{\mathrm{SHV}-11}$ & $25(74 \%)$ & $25(96 \%)$ & $21(50 \%)$ & $2(40 \%)$ & $73(68 \%)$ \\
\hline$b / a_{\mathrm{SHV}-12}$ & $6(18 \%)$ & - & - & - & $6(6 \%)$ \\
\hline$b / a_{\mathrm{SHV}-28}$ & - & - & $3(7 \%)$ & $2(40 \%)$ & $5(5 \%)$ \\
\hline$b / a_{\mathrm{SHV}-77}$ & - & - & $1(2 \%)$ & - & $1(1 \%)$ \\
\hline$b / a_{\mathrm{LEN}-16}$ & - & - & $1(2 \%)$ & - & $1(1 \%)$ \\
\hline$b / a_{L E N-27}$ & $1(3 \%)$ & - & - & - & $1(1 \%)$ \\
\hline \multicolumn{6}{|c|}{ Acquired $\beta$-lactamase genes } \\
\hline bla $a_{\mathrm{TEM}-1}$ & $25(74 \%)$ & $23(88 \%)$ & $34(81 \%)$ & $3(60 \%)$ & $85(80 \%)$ \\
\hline$b l a_{\mathrm{OXA}-1}$ & - & $1(4 \%)$ & 27 (64\%) & $2(40 \%)$ & $30(28 \%)$ \\
\hline$b / a_{\text {OXA-9 }}$ & $1(3 \%)$ & - & $10(24 \%)$ & 1 (20\%) & $12(11 \%)$ \\
\hline$b / a_{\mathrm{CTX}-\mathrm{M}-14}$ & - & - & $4(10 \%)$ & - & $4(4 \%)$ \\
\hline$b / a_{\mathrm{CTX}-\mathrm{M}-15}$ & $1(3 \%)$ & $1(4 \%)$ & 15 (36\%) & $5(100 \%)$ & $22(21 \%)$ \\
\hline$b l a_{\mathrm{CMY}-2}$ & - & - & $1(2 \%)$ & - & $1(1 \%)$ \\
\hline bla $a_{V E B-1}$ & $1(3 \%)$ & - & - & - & $1(1 \%)$ \\
\hline
\end{tabular}

\section{Content of additional $\beta$-lactamase genes}

Besides the four corresponding carbapenemase genes, a number of additional $\beta$-lactamase genes were determined from WGS data using ResFinder (Table 1). Five variants of the chromosomally encoded SHV $\beta$ lactamase were identified. SHV-11 was the most frequent subtype found in $68 \%$ of isolates. However, one OXA-48-producing and one KPC-2-producing isolate did not show any $b l a_{\mathrm{SHV}}$ gene but a bla $a_{\mathrm{LEN}}$ gene indicative of the species Klebsiella variicola. Furthermore, the majority of isolates possessed bla $a_{\mathrm{TEM}-1}$ (80\%); $28 \%$ contained bla $a_{\text {OXA-1 }}$ and $11 \%$ bla $a_{\text {OXA-9. }}$. Additional extendedspectrum $\beta$-lactamase (ESBL) genes $b l a_{\mathrm{CTX}-\mathrm{M}-15}$ and bla $a_{\mathrm{CTX}-\mathrm{M}-14}$ were found in $24 \%$ and $4 \%$ of the isolates, respectively. OXA-48- and NDM-1-producing isolates possessed an additional ESBL gene more often than KPC-2- and KPC-3-producing isolates (Table 1).

\section{Content of virulence genes}

All but one of the 107 investigated isolates contained three or less of the tested virulence genes or gene clusters. The most frequent gene cluster was mrk encoding regulation and expression of the type 3 pilus. Genes $m r k A, m r k B, m r k C, m r k D, m r k F, m r k H, m r k I$ and $m r k J$ were identified in all isolates. However, the $m r k H$ gene was interrupted by an insertion of an IS element in 29 isolates; one of which additionally showed insertion of an IS element in mrkI. Another isolate showed an 
insertion within the mrkJ gene. Correspondingly, only 77 isolates (72\%) possessed an entire mrk gene cluster (Additional file 1: Figure S2). Strikingly, in 26 of 29 strains the insertion site in $m r k H$ was completely identical. The ORF was destroyed after 207 nucleotides by the introduction of an IS element (93\% identity to ISEc21 according to ISfinder [23]). These 26 isolates were KPC2 producers of ST258 (wzi_29). Yersiniabactin was the second most frequent virulence factor. Two isolates possessed a premature STOP codon in each gene $(y b t A$ and irp2). Accordingly, $36 \%$ of all isolates harboured a complete and putatively functional yersiniabactin cluster. The $k f u$ gene cluster was identified in $21 \%(n=22)$ including all isolates of ST101 $(n=16)$ and ST15 $(n=4)$. Genes encoding the siderophore aerobactin were detected in six isolates.

The single ST23 isolate producing OXA-48 (no. 316/ 15) was exceptional by harbouring a great repertoire of virulence genes showing hits for all tested targets except $k v g$ (Additional file 3: Table S3). The ORF of rmpA2 demonstrated a premature stop codon (see also Discussion). Due to the high number of virulence genes the so-called 'string test' was performed to identify a putative hypermucoviscous phenotype associated with hypervirulence; however, the test result was repeatedly negative.

\section{Identifying the capsule type (wzi typing)}

NGS data were used to extract $w z i$ allele variants which finally deduced the corresponding capsule type [20]. Details are given in Additional file 3: Table S4. Altogether $25 w z i$ alleles were identified including five new variants that received new allele types after submission to the corresponding database at the Pasteur Institute, Paris. One isolate did not reveal any wzi result, either from analysing NGS data or from performing wzi-specific PCRs (Additional file 3: Tables S2/S4; isolate 274/15, ST147). Isolates of a single ST did not always possess the same $w z i$ allele. For instance, K. pneumoniae ST15 isolates possessed three $w z i$ alleles ( $w z i \_24, w z i \_19$ and $\left.w z i \_n e w 1\right)$. Isolates of ST147 also possessed three different allele types; however, the vast majority were associated with wzi_64. Eighty-four percent (26/31) of ST258 isolates contained the $w z i$ 29 allele, which is equivalent to capsule cluster type cps1. All these isolates also produced KPC-2. In contrast, all KPC-3-producing ST258 isolates showed wzi_154 (associated with the cps2 cluster). All isolates of ST512 $(n=21)$ contained $w z i \_154$. The single ST23 isolate no. 316/15 possessing a high number of virulence genes possessed $w z i_{-} 1$ which is associated with capsule type K1. It has been described that K. pneumoniae with capsule type $\mathrm{K} 1$ belong to the group of hypervirulent strain types [24].

\section{Plasmid content}

Using the tool PlasmidFinder the plasmid content and the equivalent incompatibility (Inc) groups were extracted from NGS data (Fig. 2). Most frequent were $\operatorname{IncFII}(\mathrm{K})$ and $\operatorname{IncFIB}(\mathrm{K})$ occurring in $76 \%$ and $68 \%$ of all isolates, respectively. All KPC-2- and KPC-3-producing isolates possessed $\operatorname{IncFII}(\mathrm{K})$ and approximately $90 \%$ also possessed $\operatorname{IncFIB}(\mathrm{K})$. Seventy-three percent of KPC-2and KPC-3-producing isolates were positive for IncX3. All NDM-1-producing isolates showed a positive result for IncFIB whereas all OXA-48-producing $K$. pneumoniae isolates possessed IncL/M (IncL/M(pMU407) or IncL/M(pOXA-48)). Furthermore, the majority of OXA48-producing isolates contained an IncR plasmid (62\%).

\section{Phylogeny of carbapenemase-producing K. pneumoniae isolates from Germany}

Searching for $\beta$-lactamase genes in NGS data revealed two isolates with a $b l a_{\mathrm{LEN}}$ gene indicative of the species K. variicola (isolates no. $275 / 15$ and no. 328/15). In silico restriction of gyrA with TaqI und HaeIII confirmed their association with phylogroup III and species $K$. variicola, respectively. Accordingly, these two isolates were not considered for subsequent phylogenetic analysis.

Phylogenetic comparison was done using a referencebased mapping approach. The best reference was selected by refRank, suggesting $K$. pneumoniae isolate KPNIH30 (NCBI-no. NZ_CP009872.1) as best match. One of the $K$. variicola isolates (ST906, OXA-48) was included to root the tree. Two K. pneumoniae isolates (no. 301/15 [bla $a_{O X A-48} ;$ ST16] and no. 330/15 [bla OXA-48; ST48]) were excluded from the mapping and tree calculation due to a high number of ambiguous sites (cutoff: $\geq 15 \%$ " $N$ " in the consensus sequence). After excluding repetitive regions and mobile genetic elements, 159,154 SNP positions (length of the reference genome: 5,306,618 bp) remained to construct the maximum likelihood tree (Fig. 3).

Among the 103 analysed carbapenemase-producing $K$. pneumoniae isolates four major clusters could be identified corresponding to sequence types ST258, ST512, ST147, and ST101. A strong association between the MLST type and carbapenemase type was noticeable for isolates of ST258, ST512, and ST101. The ST512 isolates contained KPC-3 exclusively. Furthermore, the vast majority of ST258 isolates possessed KPC-2. All but one isolates of ST101 produced OXA-48. In contrast, ST147 isolates produced mainly OXA-48, and to a lesser extent also KPC-2 and NDM-1. Isolates of ST11 and ST340 constitute a clonal group CG258 together with isolates of ST258 and ST512, a detailed view is given in Additional file 1: Figure S3. ST512 isolates formed a sub-cluster within CG/ST258 isolates. Within the CG/ST258 cluster three subgroups were noticeable which correlate with the results of the wzi typing. All isolates of ST258 associated 


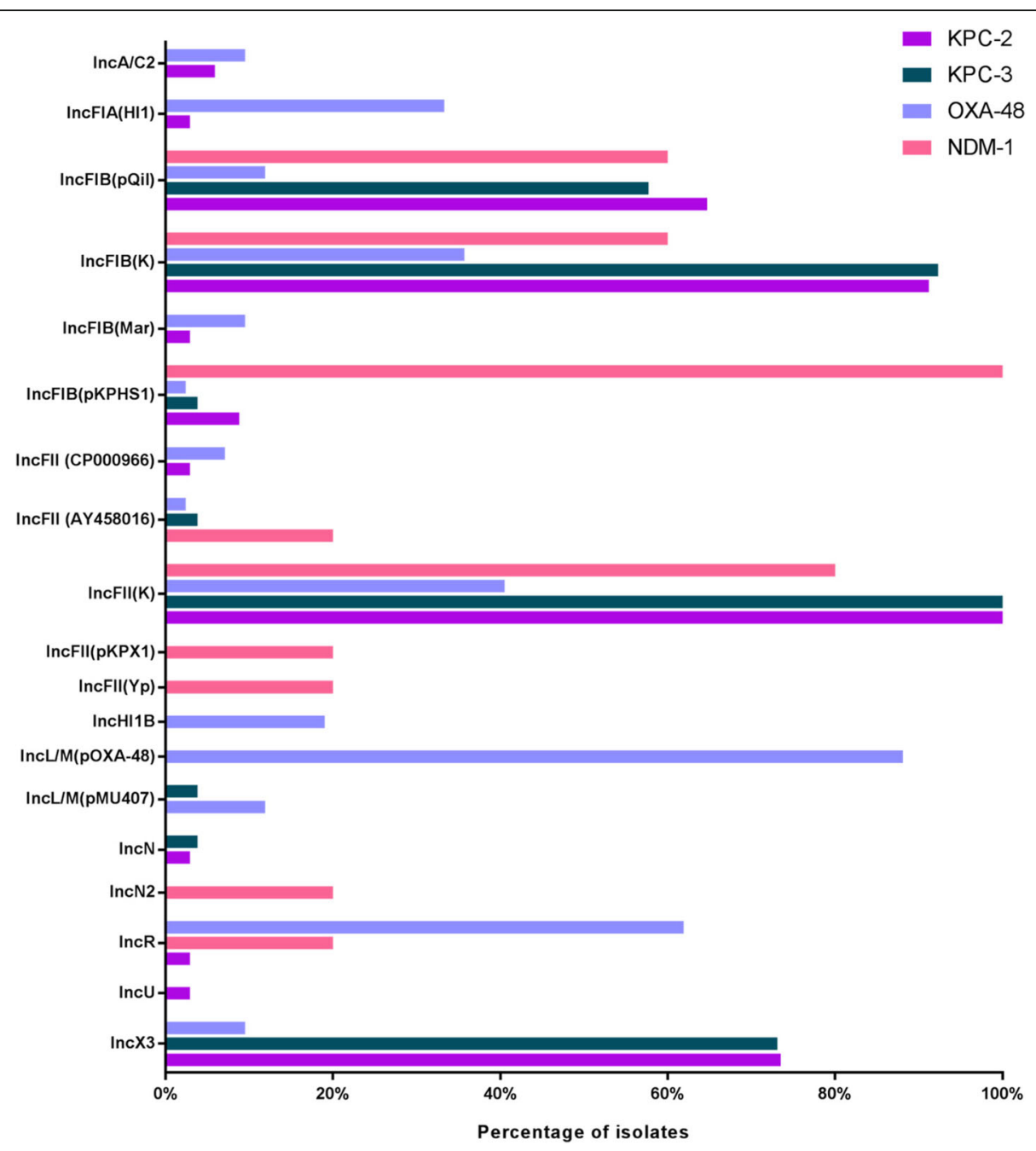

Fig. 2 Identified plasmid replicons in 107 carbapenemase-producing K. pneumoniae isolates using NGS data and PlasmidFinder software. The inc groups were presented at the $y$ axis and the color code refers to the corresponding carbapenemase genes (see legend)

with the $w z i$ 29 built a separate cluster as well as the four ST258 isolates with the wzi_154 allele. The latter were located on a separate branch and clearly different from ST512 possessing the $w z i \_154$ allele as well. One single isolate of ST258 possessed wzi_83, but was clearly separated from all other CG258 isolates. The association of the $w z i$ allele and the carbapenemase type was even stricter than with the sequence type; $w z i \_154$ was always linked to KPC-3 and wzi_29 was exclusively associated with KPC-2.

\section{Phylogeny of carbapenemase-producing K. pneumoniae} isolates from Germany in the international context

To reconstruct a phylogeny of the German carbapenemaseproducing $K$. pneumoniae isolates in relation to clinical K. pneumoniae from other countries, we performed NGS-based analysis with $K$. pneumoniae genomes from our study and two other collections (irrespective of the carbapenemase type or resistance phenotype in general): all human K. pneumoniae isolates (KpnI) described in the study by Holt et al., 2015 [25] and all completely closed K. pneumoniae genomes in the ncbi Genome Database (https://www.ncbi.nlm.nih.gov/genome/; 12/ 2016). In preparation of the analysis, completely closed genomes were artificially separated into MiSeq reads and subsequently all NGS data from MiSeq and HiSeq [25] runs were subjected to a reference-based mapping as described before but using $K$. pneumoniae subsp. pneumoniae NTUH-K2044 (NCBI-no. NC_012731. 1 ; was also used as a reference by Holt et al., [25]). Isolates with more than $15 \%$ ambiguities were excluded from subsequent analyses resulting in a tree consisting of $337 \mathrm{~K}$. pneumoniae isolates including 99 isolates from our study and 238 isolates from the other two studies (Holt collection: 167; ncbi: 71). After excluding repetitive regions and mobile genetic elements 126,347 SNPs remained to reconstruct an ML tree (Fig. 4). The majority of the other 


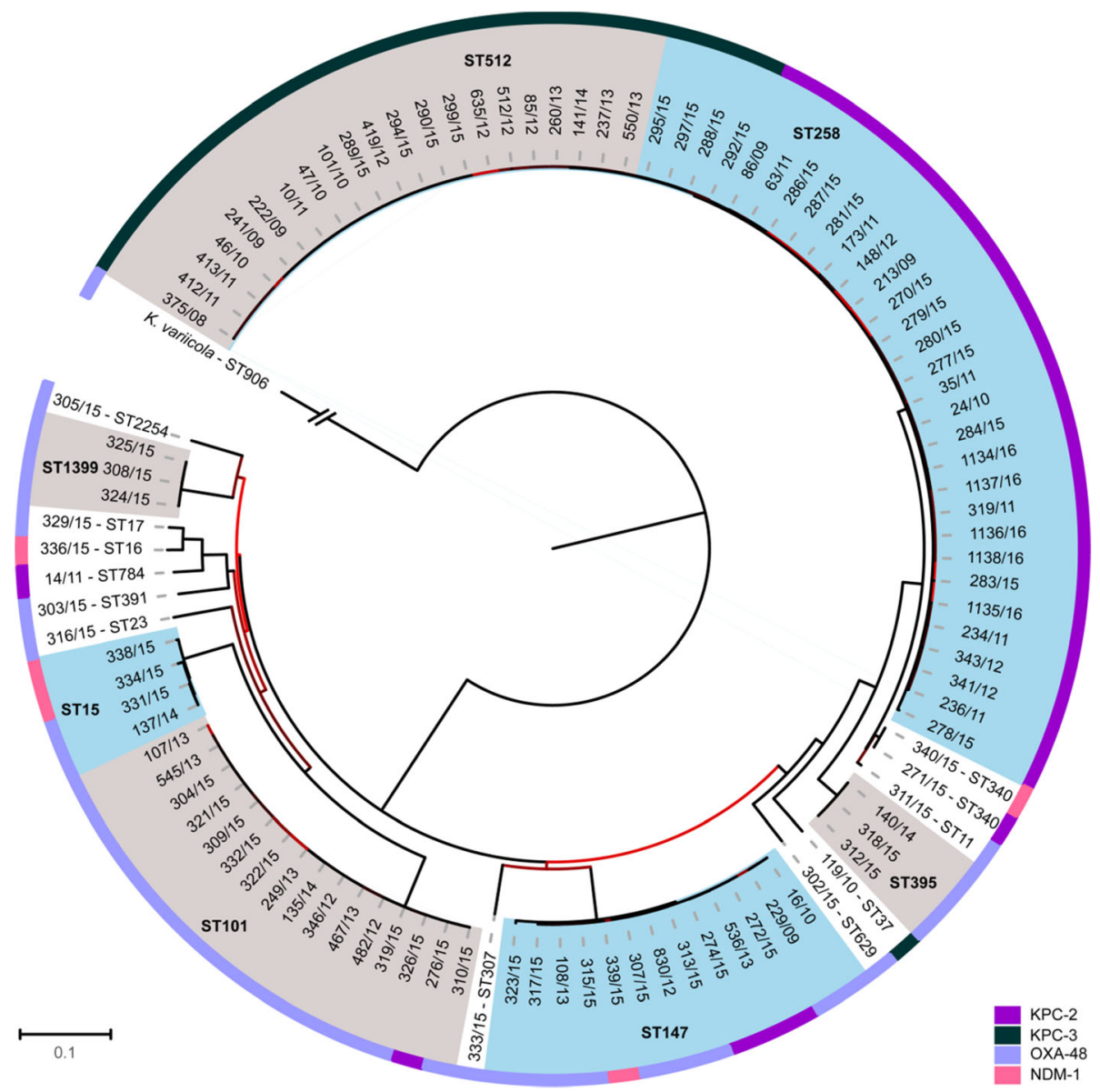

Fig. 3 Phylogeny of 103 carbapenemase-producing K. pneumoniae isolates from German hospital patients, 2008-2014. The image shows a ML tree based on 159.154 SNPs of the core genome. A K. variicola isolate (ST906, OXA-48) served as an outgroup to root the tree (the corresponding branch is shortened 1/100). Clades of three or more isolates received an alternating colour code grey/blue. The clades are labelled with the corresponding MLST type. The outer ring corresponds to the four different carbapenemase (see Figure legend for colour code). The colour of the branches correlate with the corresponding boostrap value (light red (0) to black (100))

isolates originated from Asia $(n=139)$, followed by America $(n=59)$ and Australia $(n=33)$. Furthermore, the collection included seven European isolates, three from Greece; one each from Switzerland and France and two from Germany (independent from the present collection). NGS analyses revealed 116 MLST types within the whole collection, 36 of which appeared more than once (the MLST type of 16 isolated could not be determined). The 238 international isolates comprised 108 different MLST types, 33 of which appeared more than once, demonstrating a much higher diversity than identified for the 99 isolates from this study from Germany only (21 MLST types). The largest clusters were formed by isolates belonging to ST11, ST14, ST15, ST23, ST101, ST147, ST258 and ST512. The ST14 cluster only contained isolates from international collections whereas the ST101 cluster exclusively showed isolates from Germany. Clusters ST15, ST147, ST258, and ST512 demonstrated an intermingled subset structure consisting of German and international
K. pneumoniae isolates. ST23 was the most frequent MLST type in Asia but was found only once in Germany.

We also took a closer look into the CG/ST258 cluster isolates (66 of the 337 isolates; Additional file 1: Figure S4). The majority of isolates derived from the German collection $(n=48)$, another 18 were deposited in GenBank as complete genomes and originated from America/USA $(n=17)$ and Switzerland $(n=1)$. All international ST258 and ST512 isolates also possessed a carbapenemase gene. All American isolates were of ST258 and the Swiss isolate was ST512. All but two isolates contained the $w z i+154$ or $w z i \_29$ alleles $\left(w z i \_150\right.$ in isolate NZ_CP010392.1; wzi_83 in no. 278/15). The two isolates demonstrating unique $w z i$ alleles were also distantly related to the rest. A strong association between carbapenemase type and $w z i$ allele type was identified, as already shown for the German isolates; KPC-2 was always linked to wzi_29 and KPC-3 to wzi_154. Within the ST258 group, isolates possessing KPC-2 (wzi_29) from 


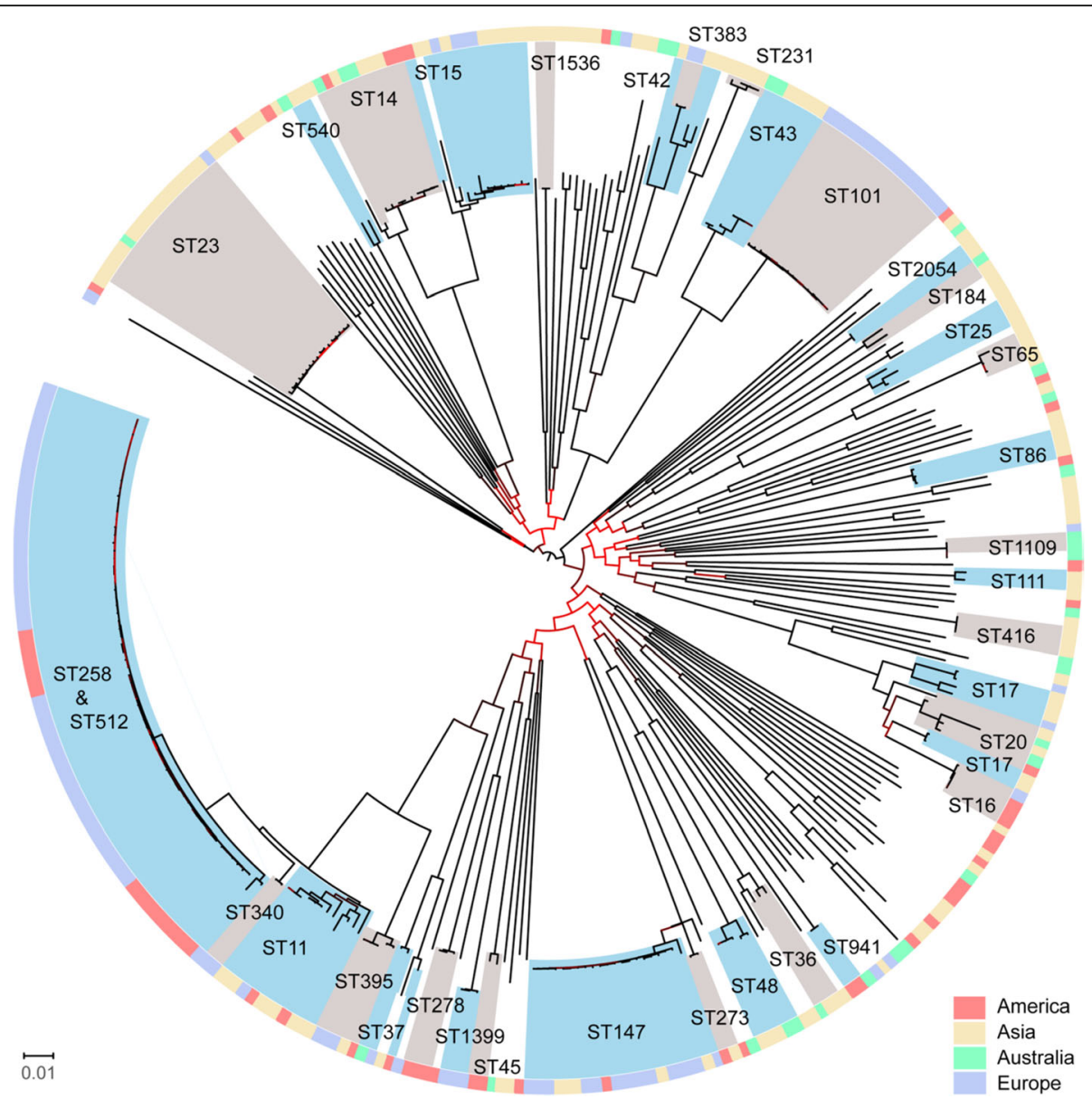

Fig. 4 Phylogeny of carbapenemase-producing isolates from Germany, 2008-2014 in relation to an international K. pneumoniae isolate collection. The image shows a ML tree based on 126.347 SNPs for 99 carbapenemase-producing isolates from Germany and 238 international K. pneumoniae isolates. The tree was rooted midpoint. Clades represented by two or more isolates were colour-coded/shaded (grey/blue) and the MLST type is listed. The outer ring corresponds to the origin of the strains given as a colour code (see Figure legend). The colours of the branches correlate with the corresponding boostrap value (light red (0) to black (100))

Germany and America formed two separate sub-clusters. The same situation was noticeable for ST258 possessing KPC-3 (wzi_154). Neither finding indicates a recent exchange of isolates between Germany and the US. In contrast, the Swiss isolate (NZ_CP015822.1) clustered together with all the German ST512 isolates.

\section{Discussion}

More than half of the study sample $(58 / 105 ; 2 \times K$. variicola) belonged to the prominent and worldwide distributed clonal group CG258, including ST258, ST512, ST11, ST395, and ST340. In collections from other countries and continents ST258 and ST11 were the most frequent sequence types within CG258 [26]. Isolates of ST258 are worldwide frequently associated with KPC-2, a finding which was also confirmed for the situation in Germany. SNP-based analyses of ST258/KPC-2 and ST512/KPC-3 showed a clear genetic separation of isolates from Europe and the United States (Additional file 1: Figure S4). This indicates a circulation of KPC clones in Europe. The first outbreak due to K. pneumoniae-ST258 with KPC2 was reported in 2008 in Germany, and the index patient had been previously hospitalized in Greece [27]. Routine screening for carbapenemase-producing K. pneumoniae is mandatory at hospital admission in Germany only as part of a risk-based adapted approach (considering certain risk factors such as having received hospital treatment in a high-risk country) and thus patients with undetected colonization may contribute to their further spread within Germany [8].

In contrast to ST258, carbapenemase-producing ST11 (CG258) isolates are quite frequent in Asia. In our collection we found only one ST11 isolate indicating that this ST has so far occurred only rarely in Germany. ST395 isolates, representing another CG258 type, appeared three times in our collection and were always associated with OXA-48. 
OXA-48-producing K. pneumoniae isolates of ST395 were also found recently in other European countries, such as France, the Netherlands and Hungary [28-32].

The present collection also contained a cluster of ST101 isolates $(n=16), 15$ of which were OXA-48 producers. Although the two external collections included in the phylogenetic analysis did not contain ST101 K. pneumoniae, the combination ST101-OXA-48 is not unique to Germany. Clinical isolates with these characteristics were described in neighbouring European countries in recent years, such as Czech Republic, France and Belgium [28, 33, 34], and also all over Europe and North Africa $[29,35]$. ST101 isolates seem to be especially distributed in Europe, and also mainly associated with OXA-48 [36]. Two further clusters were formed by ST147 and ST15 isolates producing mainly OXA-48 but also NDM-1 and/or KPC-2 (Fig. 3). Both clonal lineages of $K$. pneumoniae are well known as colonizers or the cause of infection in human patients; outbreaks due to ST147 with OXA-48 have been reported in Germany and ST15 with OXA-48 was also found in pets in a German veterinary clinic $[37,38]$.

Due to the presence of the $b l a_{\mathrm{LEN}}$ gene and the gyrA sequence two of the 107 isolates were classified as K. variicola (phylogroup Kpn III). The same MLST scheme is used for all three phylogroups and all species $K$. variicola, K. pneumoniae and Klebsiella quasipneumoniae, respectively [36]. The OXA-48 producing $K$. variicola isolate of ST906 represents a very rare ST and ST/carbapenemase combination. ST906 was first defined in a set of strains from an Israeli clinic [39]. However, the authors failed to proof the species $K$. variicola. $K$. variicola isolates of ST347 were described in previous studies from China, Japan, Greece and Sweden [40-43]. However, only the Greek isolate was a carbapenemase producer (KPC-2) [42]. In general, reports about carbapenemase-producing $K$. variicola are rare; we found a report about OXA-181producing $K$. variicola from vegetables imported from Asia to Europe [44] and NDM-producing isolates originating from a river in China [45]. In a very recent study performed in the US an ST906-isolate producing NDM-1 was also identified as K. variicola [46].

One isolate was clearly separated from the rest of our collection regarding the presence of virulence genes: the OXA-48-producing ST23 isolate no. 316/15, carrying capsule type $\mathrm{K} 1$ and containing all but one tested virulence markers including different siderophore genes (Additional file 3: Table S3). ST23 isolates are known to be hypervirulent and can cause severe infections in apparently healthy individuals [47, 48]. In particular, ST23 isolates expressing the $\mathrm{K} 1$ capsule were frequently associated with cases of community-acquired pyogenic liver abscesses and occurred predominantly in Asian countries [24, 48]. Our single ST23 isolate originated from a tracheal secretion suggesting a respiratory tract infection. Subsequent molecular analyses

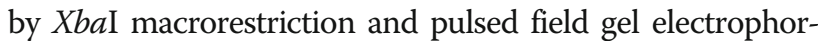
esis of isolates from the same diagnostic laboratory revealed that this isolate belonged to a cluster of seven isolates that occurred in the period February 2012 to July 2012. The sample isolates originated from three blood cultures, one urine sample and several swabs. No further clinical or patient information was available for these isolates. Hypervirulent strains of $K$. pneumoniae often express a typical hypermucoviscous phenotype and demonstrate a small spectrum of resistances, mainly only to penicillins, e.g. ampicillin. Our ST23 isolate contained various $\beta$-lactamase genes, including classical $\beta$-lactamases $\left(b l a_{\mathrm{SHV}-11}, b l a_{\mathrm{TEM}-1}\right.$, bla $\left.a_{\text {OXA-1 }}\right)$, ESBLs $\left(b l a_{\text {CTX-M-15 }}\right)$ and carbapenemases $\left(b l a_{\text {OXA-48 }}\right)$. A quick, so-called 'string test' for identifying a hypermucoviscous phenotype was negative, possibly associated with the premature STOP codon in the rmpA2 gene (regulator of the mucoid phenotype A gene). However, the string test gives only a hint and is not reliable enough to predict a corresponding clinical behaviour. In addition, we do not know whether this mutation was also prevalent in the other clonally related isolates that were not available for a genome-based analysis. The combination of hypervirulence and resistance to last-line therapeutics is highly worrisome and has just recently raised serious public health concerns [49-51].

A number of recent reports have suggested that the wzi locus is frequently exchanged between K. pneumoniae isolates $[52,53]$ and, as such, could be putatively genetically linked to other, horizontally exchanged markers, such as plasmid-located carbapenemase genes. In particular, the highly successful ST258 clone seemed to be a hybrid strain with the cps locus (containing the wzi gene) located next to a recombination hotspot [54]. Although KPC-2/3, OXA-48 and NDM-1 genes are commonly located on plasmids, a chromosomal integration is also reasonable, at least for some carbapenemase genes. It has been described recently, that $b l a_{\mathrm{KPC}-2}$ could also be chromosomally determined via IS-mediated Tn4401 transposition into specific chromosomal loci, especially observed in CG258 isolates (ST11, ST258, ST340) [55]. Although we could not properly elucidate plasmid structures by assembling Miseq reads, we suggest a plasmid localization of the carbapenemase genes in most cases based on the sum of assembled and interpreted NGS data. The finding of IncFII sequences in KPC-producing CG258 isolates and IncL/M sequences in all OXA-48 producers supports our suggestion because these associations of carbapenease genes and plasmid replicon types are well known $[53,56]$.

ST258 isolates (our collection and genomes of international isolates included in the study) contained four $w z i$ alleles; $w z i \_29(n=26) ; w z i \_154(n=4) ; w z i \_83(n=1)$ and wzi_150 $(n=1)$. Similar distributions were described in two recent studies performed in the US [52, 57] The 
association within ST258 isolates of cps1/wzi_29 with $b l a_{\mathrm{KPC}-2}$ and $c p s 2 / w z i \_154$ with $b l a_{\mathrm{KPC}-3}$, respectively, was identified for our study sample of German clinical isolates but was also described for isolates from other studies and countries $[54,58,59]$. Bowers et al., supposed that the association of cps 1 in KPC-2-producing K. pneumoniae isolates and of cps 2 in KPC-3-positive isolates is derived from the fact that the point mutations in $b l a_{\mathrm{KPC}}$ leading to a differentiation into $b l a_{\mathrm{KPC}-2}$ and $b l a_{\mathrm{KPC}-3}$ appeared at the same time when the two clades with different cps loci diverged [58]. All of the ST512 isolates in our study contained $b l a_{\mathrm{KPC}-3}$ and $w z i-154$; which is identical to all

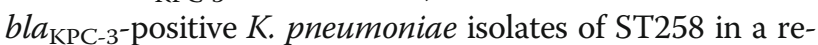
cent study performed in Italy. Thus, our results presented for German isolates confirm previous findings performed

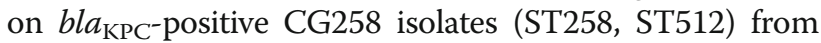
Italy [59]. In all ST512 isolates from Italy only wzi_154 was present. This exclusive association among a comprehensive set of $461 \mathrm{~K}$. pneumoniae isolates collected all over the country led the authors of the previous study to assume that ST512 isolates may have originated from ST258-cps2-clade strains [59].

\section{Limitations}

Our study has some limitations. First, strain sending to reference laboratories is voluntary and taking this into consideration we cannot exclude any bias in our strain collection, which served as the basis to select representative isolates for further genome-based analysis. Second, clinical and patient data available with the strains submitted were limited. We could not perform any analysis in this regard, nor could we provide additional clinical and patient information to the data that are already provided. Third, we are well aware that even a collection of about 100 strains representing 12 federal states in Germany and a timeframe of 7 years does not allow statistically significant assumptions (which we have not calculated and specified as such). Fourth, it has already been addressed in previous papers that plasmid content is difficult to reconstruct with Miseq data, thus the location of the carbapenemase gene on a specific plasmid could and, in consequence, a potential spread of distinct carbapenemase gene-carrying plasmids not be identified. Correspondingly, the putative link between plasmid types and carbapenemase genes remains to a certain extent hypothetical. Fifth, in the last 2 years, data from the NRC showed a rapid increase of Enterobacteriaceae (including K. pneumoniae) isolates producing VIM-1 in Germany [2], but this trend could not be considered when this study was initiated.

\section{Conclusion}

Using WGS-based analysis we were able to describe a population snapshot of clinical, carbapenemase-producing $K$. pneumoniae isolates from hospitalized patients in Germany collected between 2008 and 2014. This revealed a preferred prevalence of KPC-2-producing ST258 isolates and KPC-3-producing ST512 isolates. OXA-48 is the most prevalent carbapenemase type in Germany and was shown to be associated with various $K$. pneumoniae strain types, most of them possessing IncL/M plasmid replicons, suggesting a preferred dissemination of $b l a_{\mathrm{OXA}-48}$ via this well-known plasmid type. We identified a supposed hypervirulent ST23 strain of capsule type K1-producing OXA48 , which turned out to be representative for an entire cluster of infections in a single German health-care centre in 2012. This finding demonstrated that this highly pathogenic and multidrug-resistant $K$. pneumoniae strain type, associated with a serious public health threat outside Asia and within Europe, had already appeared several years ago.

\section{Additional files}

Additional file 1: Figure S1. Origin of the 107 German carbapenemaseproducing K. pneumoniae isolates. Regions are shown, where isolates originated from. Isolates from Saxony could not be elucidated further due to the lack of additional geographic information. Number of isolates is given by the size of the circle (see legend). Image is from: @ Bundesamt für Kartographie und Geodäsie, Frankfurt am Main, Germany. Figure S2. Virulence gene content in 107 carbapenemase-producing K. pneumonaie isolates from Germany. Data are given in \% of isolates showing possession of the corresponding gene cluster. The graph shows four most frequent virulence genes identified in more than one single isolate. Figure S3. Detailed view of the ML tree concerning ST258/ST512 - carbapenemase-producing K. pneumoniae isolates from Germany, 2008-2014. The image shows a subtree of Fig. 3 containing 52 isolates of ST258 (light violett) and ST512 (grey). Colour codes of the inner ring designate the corresponding carbapenemase type, the outer designates the wzi allele (see legend). Figure S4. ML tree of NGS-based analysis of German K. pneumoniae isolates and isolates from an international collection - detailed view of the cluster ST258/ST512 isolates. The image shows a subtree of Fig. 4 containing 66 isolates of ST258 and ST512. Colour codes of the inner ring correspond to the origin of strains, the middle ring to the carbapenemase KPC-2 or KPC-3, and the outer ring demonstrates the wzi allele type. (PPTX $1367 \mathrm{~kb}$ )

Additional file 2: Table S1. Metadata of the 107 German carbapenemase-producing, clinical Klebsiella isolates, 2008-2014. Isolates were ordered by carbapenemase type. For more information see main text document. (XLSX 54 kb)

Additional file 3: Table S2. Names and sequences of used primers for MLST and PCR amplification of virulence genes and $\beta$-lactamase genes. Table S3. Identified virulence genes in K. pneumoniae isolate no. 316/15 (ST23, OXA-48). Table S4. Identified wzi alleles from NGS data of 107 carbapenemase-producing K. pneumoniae from Germany. (DOCX 35 kb)

\section{Acknowledgements}

We thank Kirstin Ganske for excellent technical assistance. We thank the team of the curators of the Institut Pasteur MLST system (Paris, France) for importing novel alleles, profiles and/or isolates at http://bigsdb.web.pasteur.fr. A special thanks to Prof. Dr. Sylvain Brisse, Pasteur Institute, Paris, for the possibility of a scientific exchange on selected subjects of the project. We thank Prof. Dr. Ulrich Nübel, DSMZ Braunchweig, Germany, for his contributions to the project application (see above) and for helpful discussions.

\section{Funding}

This project has received funding from the German Federal Ministry of Health (IIA5-2513NIK006/321-4471-02/129).

Availability of data and materials

Sequence data were submitted to the European Nucleotide Archive (http:/) www.ebi.ac.uk/ena) and are available under study accession number ERP020566. 


\section{Authors' contributions}

GW, SG and YP designed and supervised the study. MK and MKB did primary and confirmatory diagnostics for carbapenemase gene presence, species identification and typing by macrorestriction analysis. GW, YP, MK, MKB and SG selected carbapenemase-producing K. pneumoniae isolates for WGS. LB prepared the samples for WGS, performed the data analyses and most of the molecular experiments. SF provided several tools for WGS data analyses. AR, AvL, and MAS provided unpublished data from the country-wide notification of carbapenemresistant Enterobacteriaceae including Klebsiella. GW wrote the manuscript. All authors reviewed the manuscript. All authors read and approved the final manuscript.

\section{Ethics approval and consent to participate}

Not applicable.

\section{Competing interests}

The authors declare that they have no competing interests.

\section{Publisher's Note}

Springer Nature remains neutral with regard to jurisdictional claims in published maps and institutional affiliations.

\section{Author details}

${ }^{1}$ Robert Koch Institute, Berlin, Germany. ${ }^{2}$ National Reference Centre for Multidrug-resistant Gram-negative Bacteria, Department for Medical Microbiology, Ruhr-University Bochum, Bochum, Germany. ${ }^{3}$ Present address: Department of Infection Control and Infectious Diseases, University Medical Centre Goettingen, Goettingen, Germany. ${ }^{4}$ Fachgruppe Infektiologie und Hygiene, Landeszentrum Gesundheit North-Rhine Westphalia, Gesundheitscampus 10, Bochum, Germany.

\section{Received: 2 March 2018 Accepted: 19 April 2018}

\section{Published online: 02 May 2018}

\section{References}

1. ECDC. Antimicrobial resistance surveillance in Europe 2016; 5. Annual report of the European antimicrobial resistance surveillance network (EARS-net). In: Annual report of the European antimicrobial resistance surveillance network (EARS-net). Stockholm: European Centre for Disease Prevention and Control (ECDC); 2017.

2. Pfennigwerth $\mathrm{N}$. Report of the National Reference Centre for gram-negative nosocomial pathogens, 2016. Epidemiologisches Bull. 2017;26:5.

3. Kaase M, Pfennigwerth $\mathrm{N}$. Report from the National Reference Centre for gram-negative nosocomial pathogens, 2015. Epidemiologisches Bull. 2016; 41(25):5.

4. Kaiser T, Finstermeier K, Hantzsch M, Faucheux S, Kaase M, Eckmanns T, Bercker S, Kaisers UX, Lippmann N, Rodloff AC, et al. Stalking a lethal superbug by whole-genome sequencing and phylogenetics: influence on unraveling a major hospital outbreak of carbapenem-resistant Klebsiella pneumoniae. Am J Infect Control. 2017;46(1):54-59. https://doi.org/10.1016/j. ajic.2017.07.022

5. Carstens A, Kepper U, Exner M, Hauri AM, Kaase M, Wendt C. Plasmidmediated multispecies outbreak with carbapenemase-resistant Enterobacteriaceae. Epidemiologisches Bull. 2014:47:5.

6. Kola A, Piening B, Pape UF, Veltzke-Schlieker W, Kaase M, Geffers C, Wiedenmann B, Gastmeier P. An outbreak of carbapenem-resistant OXA-48producing Klebsiella pneumoniae associated to duodenoscopy. Antimicrob Resist Infect Control. 2015:4:8.

7. Steinmann J, Kaase M, Gatermann S, Popp W, Steinmann E, Damman M, Paul A, Saner F, Buer J, Rath P. Outbreak due to a Klebsiella pneumoniae strain harbouring KPC-2 and VIM-1 in a German university hospital, July 2010 to January 2011. Euro Surveill. 2011;16(33.

8. Kaase M, Schimanski S, Schiller R, Beyreiss B, Thurmer A, Steinmann J, Kempf VA, Hess C, Sobottka I, Fenner I, et al. Multicentre investigation of carbapenemase-producing Escherichia coli and Klebsiella pneumoniae in German hospitals. Int J Med Microbiol. 2016;306(6):415-20.

9. Bolger AM, Lohse $M$, Usadel B. Trimmomatic: a flexible trimmer for Illumina sequence data. Bioinformatics. 2014;30(15):2114-20.

10. Li H, Durbin R. Fast and accurate short read alignment with burrowswheeler transform. Bioinformatics. 2009;25(14):1754-60.
11. Koboldt DC, Zhang Q, Larson DE, Shen D, McLellan MD, Lin L, Miller CA Mardis ER, Ding L, Wilson RK. VarScan 2: somatic mutation and copy number alteration discovery in cancer by exome sequencing. Genome Res. 2012:22(3):568-76.

12. Stamatakis A. RAxML version 8: a tool for phylogenetic analysis and postanalysis of large phylogenies. Bioinformatics. 2014;30(9):1312-3.

13. Huang W, Li L, Myers JR, Marth GT. ART: a next-generation sequencing read simulator. Bioinformatics. 2012;28(4):593-4.

14. Li H, Handsaker B, Wysoker A, Fennell T, Ruan J, Homer N, Marth G, Abecasis G, Durbin R, Genome Project Data Processing S. The sequence alignment/ map format and SAMtools. Bioinformatics. 2009;25(16):2078-9.

15. Becker L, Fuchs S, Pfeifer $Y$, Semmler T, Eckmanns T, Korr G, Sissolak D, Friedrichs $M$, Zill E, Tung M-L et al: Whole genome sequence analysis of CTX-M-15 producing Klebsiella isolates allowed dissecting a polyclonal outbreak scenario. Front Microbiol 2018, (online first):14.

16. Arndt D, Grant JR, Marcu A, Sajed T, Pon A, Liang Y, Wishart DS. PHASTER: a better, faster version of the PHAST phage search tool. Nucleic Acids Res. 2016;44(W1):W16-21.

17. Coil D, Jospin G, Darling AE. A5-miseq: an updated pipeline to assemble microbial genomes from Illumina MiSeq data. Bioinformatics. 2015;31(4):587-9.

18. Zankari E, Hasman H, Cosentino S, Vestergaard M, Rasmussen S, Lund O, Aarestrup FM, Larsen MV. Identification of acquired antimicrobial resistance genes. J Antimicrob Chemother. 2012;67(11):2640-4.

19. Larsen MV, Cosentino S, Rasmussen S, Friis C, Hasman H, Marvig RL, Jelsbak L, Sicheritz-Ponten T, Ussery DW, Aarestrup FM, et al. Multilocus sequence typing of total-genome-sequenced bacteria. J Clin Microbiol. 2012;50(4):1355-61.

20. Brisse S, Passet V, Haugaard AB, Babosan A, Kassis-Chikhani N, Struve C, Decre D. wzi gene sequencing, a rapid method for determination of capsular type for Klebsiella strains. J Clin Microbiol. 2013;51(12):4073-8.

21. Kumabe A, Kenzaka T. String test of hypervirulent Klebsiella pneumonia. QJM. 2014;107(12):1053.

22. Pichler C, Buchsel M, Rossen JW, Vavra M, Reuter S, Kern WV, Thimme R, Mischnik A. First report of invasive liver abscess syndrome with endophthalmitis caused by a K2 serotype ST2398 hypervirulent Klebsiella pneumoniae in Germany, 2016. New Microbes New Infect. 2017;17:77-80.

23. Siguier P, Perochon J, Lestrade L, Mahillon J, Chandler M. ISfinder: the reference Centre for bacterial insertion sequences. Nucleic Acids Res. 2006; 34(Database issue):D32-6.

24. Struve C, Roe CC, Stegger M, Stahlhut SG, Hansen DS, Engelthaler DM, Andersen PS, Driebe EM, Keim P, Krogfelt KA. Mapping the evolution of Hypervirulent Klebsiella pneumoniae. MBio. 2015;6(4):e00630.

25. Holt KE, Wertheim H, Zadoks RN, Baker S, Whitehouse CA, Dance D, Jenney A, Connor TR, Hsu LY, Severin J, et al. Genomic analysis of diversity, population structure, virulence, and antimicrobial resistance in Klebsiella pneumoniae, an urgent threat to public health. Proc Natl Acad Sci U S A. 2015;112(27):E3574-81.

26. Deleo FR, Chen L, Porcella SF, Martens CA, Kobayashi SD, Porter AR, Chavda KD, Jacobs MR, Mathema B, Olsen RJ, et al. Molecular dissection of the evolution of carbapenem-resistant multilocus sequence type 258 Klebsiella pneumoniae. Proc Natl Acad Sci U S A. 2014;111(13):4988-93.

27. Wendt C, Schutt S, Dalpke AH, Konrad M, Mieth M, Trierweiler-Hauke B, Weigand MA, Zimmermann S, Biehler K, Jonas D. First outbreak of Klebsiella pneumoniae carbapenemase (KPC)-producing K. pneumoniae in Germany. Eur J Clin Microbiol Infect Dis. 2010;29(5):563-70.

28. Liapis E, Pantel A, Robert J, Nicolas-Chanoine $\mathrm{MH}$, Cavalie L, van der Mee-Marquet N, de Champs C, Aissa N, Eloy C, Blanc V, et al. Molecular epidemiology of OXA-48-producing Klebsiella pneumoniae in France. Clin Microbiol Infect. 2014;20(12):01121-3.

29. Potron A, Poirel $L$, Rondinaud $E$, Nordmann P. Intercontinental spread of OXA-48 beta-lactamase-producing Enterobacteriaceae over a 11-year period, 2001 to 2011. Euro Surveill. 2013;18(31).

30. Cuzon G, Ouanich J, Gondret R, Naas T, Nordmann P. Outbreak of OXA-48positive carbapenem-resistant Klebsiella pneumoniae isolates in France. Antimicrob Agents Chemother. 2011:55(5):2420-3.

31. Kovacs K, Nyul A, Mestyan G, Melegh S, Fenyvesi H, Jakab G, Szabo H, Janvari L, Damjanova I, Toth A. Emergence and interhospital spread of OXA48-producing Klebsiella pneumoniae ST395 clone in western Hungary. Infect Dis (Lond). 2017:49(3):231-3.

32. Potron A, Kalpoe J, Poirel L, Nordmann P. European dissemination of a single OXA-48-producing Klebsiella pneumoniae clone. Clin Microbiol Infect. 2011;17(12):E24-6. 
33. De Laveleye $M$, Huang TD, Bogaerts $P$, Berhin C, Bauraing C, Sacre P, Noel A, Glupczynski Y, multicenter study g. Increasing incidence of carbapenemaseproducing Escherichia coli and Klebsiella pneumoniae in Belgian hospitals. Eur J Clin Microbiol Infect Dis. 2017;36(1):139-46.

34. Skalova A, Chudejova K, Rotova V, Medvecky M, Studentova V, Chudackova E, Lavicka P, Bergerova T, Jakubu V, Zemlickova H, et al. Molecular characterization of OXA-48-like-producing Enterobacteriaceae in the Czech Republic and evidence for horizontal transfer of pOXA-48-like plasmids. Antimicrob Agents Chemother. 2017;61(2):e01889-16.

35. Canton R, Akova M, Carmeli Y, Giske CG, Glupczynski Y, Gniadkowski M, Livermore DM, Miriagou V, Naas T, Rossolini GM, et al. Rapid evolution and spread of carbapenemases among Enterobacteriaceae in Europe. Clin Microbiol Infect. 2012;18(5):413-31.

36. Wyres KL, Holt KE. Klebsiella pneumoniae population genomics and antimicrobial-resistant clones. Trends Microbiol. 2016;24(12):944-56.

37. Zautner AE, Bunk B, Pfeifer Y, Sproer C, Reichard U, Eiffert H, Scheithauer S, Gross U, Overmann J, Bohne W. Monitoring microevolution of OXA-48producing Klebsiella pneumoniae ST147 in a hospital setting by SMRT sequencing. J Antimicrob Chemother. 2017;72(10):2737-44

38. Stolle I, Prenger-Berninghoff E, Stamm I, Scheufen S, Hassdenteufel E, Guenther S, Bethe A, Pfeifer Y, Ewers C. Emergence of OXA-48 carbapenemase-producing Escherichia coli and Klebsiella pneumoniae in dogs. J Antimicrob Chemother. 2013;68(12):2802-8.

39. Baraniak A, Izdebski R, Fiett J, Sadowy E, Adler A, Kazma M, Salomon J, Lawrence C, Rossini A, Salvia A, et al. Comparative population analysis of Klebsiella pneumoniae strains with extended-spectrum beta-lactamases colonizing patients in rehabilitation centers in four countries. Antimicrob Agents Chemother. 2013;57(4):1992-7.

40. Ito R, Shindo Y, Kobayashi D, Ando M, Jin W, Wachino J, Yamada K, Kimura K, Yagi T, Hasegawa Y, et al. Molecular epidemiological characteristics of Klebsiella pneumoniae associated with bacteremia among patients with pneumonia. J Clin Microbiol. 2015;53(3):879-86.

41. Maatallah M, Vading M, Kabir MH, Bakhrouf A, Kalin M, Naucler P, Brisse S, Giske CG. Klebsiella variicola is a frequent cause of bloodstream infection in the Stockholm area, and associated with higher mortality compared to $K$. pneumoniae. PLoS One. 2014;9(11):e113539.

42. Pantelidou IM, Galani I, Georgitsi M, Daikos GL, Giamarellos-Bourboulis EJ. Interactions of Klebsiella pneumoniae with the innate immune system vary in relation to clone and resistance phenotype. Antimicrob Agents Chemother. 2015;59(11):7036-43.

43. Xiao SZ, Wang S, Wu WM, Zhao SY, Gu FF, Ni YX, Guo XK, Qu JM, Han LZ. The resistance phenotype and molecular epidemiology of Klebsiella pneumoniae in bloodstream infections in shanghai, China, 2012-2015. Front Microbiol. 2017:8:250.

44. Zurfluh K, Poirel L, Nordmann P, Klumpp J, Stephan R. First detection of Klebsiella variicola producing OXA-181 carbapenemase in fresh vegetable imported from Asia to Switzerland. Antimicrob Resist Infect Control. 2015:4:38.

45. Di DY, Jang J, Unno T, Hur HG. Emergence of Klebsiella variicola positive for NDM-9, a variant of New Delhi metallo-beta-lactamase, in an urban river in South Korea. J Antimicrob Chemother. 2017;72(4):1063-7.

46. Long SW, Linson SE, Ojeda Saavedra M, Cantu C, Davis JJ, Brettin T, Olsen RJ. Whole-genome sequencing of human clinical Klebsiella pneumoniae isolates reveals misidentification and misunderstandings of Klebsiella pneumoniae, Klebsiella variicola, and Klebsiella quasipneumoniae. mSphere. 2017;2(4):e00290-17.

47. Cubero M, Grau I, Tubau F, Pallares R, Dominguez MA, Linares J, Ardanuy C. Hypervirulent Klebsiella pneumoniae clones causing bacteraemia in adults in a teaching hospital in Barcelona, Spain (2007-2013). Clin Microbiol Infect. 2016;22(2):154-60.

48. Shon AS, Bajwa RP, Russo TA. Hypervirulent (hypermucoviscous) Klebsiella pneumoniae: a new and dangerous breed. Virulence. 2013;4(2):107-18.

49. Gu D, Dong N, Zheng Z, Lin D, Huang M, Wang L, Chan EW, Shu L, Yu J, Zhang R, et al. A fatal outbreak of ST11 carbapenem-resistant hypervirulent Klebsiella pneumoniae in a Chinese hospital: a molecular epidemiological study. Lancet Infect Dis. 2017;

50. Wei DD, Wan LG, Deng Q, Liu Y. Emergence of KPC-producing Klebsiella pneumoniae hypervirulent clone of capsular serotype K1 that belongs to sequence type 11 in mainland China. Diagn Microbiol Infect Dis. 2016; 85(2):192-4.

51. Yao B, Xiao X, Wang F, Zhou L, Zhang X, Zhang J. Clinical and molecular characteristics of multi-clone carbapenem-resistant hypervirulent (hypermucoviscous) Klebsiella pneumoniae isolates in a tertiary hospital in Beijing, China. Int J Infect Dis. 2015;37:107-12.

52. Diago-Navarro E, Chen L, Passet V, Burack S, Ulacia-Hernando A, Kodiyanplakkal RP, Levi MH, Brisse S, Kreiswirth BN, Fries BC. Carbapenemresistant Klebsiella pneumoniae exhibit variability in capsular polysaccharide and capsule associated virulence traits. J Infect Dis. 2014;210(5):803-13.

53. Wyres KL, Gorrie C, Edwards DJ, Wertheim HF, Hsu LY, Van Kinh N, Zadoks R, Baker S, Holt KE. Extensive capsule locus variation and large-scale genomic recombination within the Klebsiella pneumoniae clonal group 258. Genome Biol Evol. 2015;7(5):1267-79.

54. Chen L, Mathema B, Pitout JD, DeLeo FR, Kreiswirth BN. Epidemic Klebsiella pneumoniae ST258 is a hybrid strain. MBio. 2014;5(3):e01355-14.

55. Mathers AJ, Stoesser N, Chai W, Carroll J, Barry K, Cherunvanky A, Sebra R, Kasarskis A, Peto TE, Walker AS, et al. Chromosomal integration of the Klebsiella pneumoniae carbapenemase gene, blaKPC, in Klebsiella species is elusive but not rare. Antimicrob Agents Chemother. 2017;61(3):e01823-16.

56. Pfeifer $Y$, Schlatterer $K$, Engelmann E, Schiller RA, Frangenberg HR, Stiewe D, Holfelder M, Witte W, Nordmann P, Poirel L. Emergence of OXA-48-type carbapenemase-producing Enterobacteriaceae in German hospitals. Antimicrob Agents Chemother. 2012;56(4):2125-8.

57. Gomez-Simmonds A, Greenman M, Sullivan SB, Tanner JP, Sowash MG, Whittier S, Uhlemann AC. Population structure of Klebsiella pneumoniae causing bloodstream infections at a New York City tertiary care hospital: diversification of multidrug-resistant isolates. J Clin Microbiol. 2015;53(7):2060-7.

58. Bowers JR, Kitchel B, Driebe EM, MacCannell DR, Roe C, Lemmer D, de Man T, Rasheed JK, Engelthaler DM, Keim P, et al. Genomic analysis of the emergence and rapid global dissemination of the clonal group 258 Klebsiella pneumoniae pandemic. PLoS One. 2015;10(7):e0133727.

59. Conte V, Monaco M, Giani T, D'Ancona F, Moro ML, Arena F, D'Andrea MM, Rossolini GM, Pantosti A, pneumoniae A-ISGoC-PK. Molecular epidemiology of KPC-producing Klebsiella pneumoniae from invasive infections in Italy: increasing diversity with predominance of the ST512 clade II sublineage. J Antimicrob Chemother. 2016;71(12):3386-91.

\section{Ready to submit your research? Choose BMC and benefit from:}

- fast, convenient online submission

- thorough peer review by experienced researchers in your field

- rapid publication on acceptance

- support for research data, including large and complex data types

- gold Open Access which fosters wider collaboration and increased citations

- maximum visibility for your research: over $100 \mathrm{M}$ website views per year

At BMC, research is always in progress.

Learn more biomedcentral.com/submissions 